\title{
¡Películas de narcos en las aulas! Prácticas alternativas de enseñanza de la historia en Bogotá
}

\author{
Doi: https://doi.org/10.25100/hye.v15i53.8737 \\ Artículo recibido: 28-12-2018 Artículo aceptado: 06-11-2019
}

\section{Wilson Armando Acosta Jiménez}

Es Licenciado en Ciencias Sociales de la Universidad Distrital Francisco José de Caldas, Magíster en Sociología de la Educación de la Universidad Pedagógica Nacional y Doctor del Doctorado Interinstitucional en Educación de la UPN - UDFJC - U. del Valle. Es docente de planta del Departamento de Ciencias Sociales - Facultad de Humanidades, en donde dicta seminarios como: Historia y Epistemología de la Pedagogía; Didáctica de la historia, Memorias, territorios e identidades; Sociedades originarias del mundo; Problemas actuales de américa latina Universidad Pedagógica Nacional, Colombia.

Correo electrónico: wacosta@pedagogica.edu.co

ORCID: 0000-0003-1411-7341

Forma de citar este artículo: Acosta Jiménez, Wilson Armando. "Películas de narcos en las aulas! Prácticas alternativas de enseñanza de la historia en Bogotá". Historia y Espacio, vol. 15 n 53 (2019): 221-248. Doi.org/10.25100/hye.v15i53.8737.

Artículo Tipo 1: de investigación. 


\section{¡Películas de narcos en las aulas! Prácticas alternativas de enseñanza de la historia en \\ Bogotá}

Resumen: El objetivo de este artículo es exponer un análisis desde la didáctica de la historia sobre la irrupción del cine sobre narcos en las prácticas de enseñanza de la historia en Bogotá. Se desarrolla en tres partes: en la primera, se presenta la rejilla de análisis y las condiciones del estudio. En la segunda parte, se caracterizan las experiencias de los profesores a partir de los ámbitos de problematización y las categorías de análisis. Y, en la tercera, se identifican las rupturas en las prácticas de enseñanza de las versiones del tiempo presente que acontecen en el aula.

Palabras clave: Análisis del discurso, Historia escolar, Prácticas de enseñanza, Dispositivo didáctico y código disciplinar.

\section{Narco movies in the classrooms! Alternatives teaching practices of history in Bogotá}

Abstract: The objective of this article is to present an analysis from the didactics of history about the irruption of cinema about "narcos" in the teaching practices of history in Bogotá. This is developed in three parts: in the first, the analysis grid and the conditions of the study are presented. In the second part the experiences of the professors are characterized from the areas of problematization and the categories of analysis. And in the third, the ruptures are identified in the teaching practices of the present time versions that take place in the classroom.

Keywords: Discourse analysis, School history, Teaching practices, Didactic device and disciplinary code. 


\section{Wilson Armando Acosta Jiménez}

\section{¡Películas de narcos en las aulas! Prácticas alternativas de enseñanza de la historia en

\section{Introducción}

En una era posliteraria, ya no habrá necesidad de periódicos y libros. Los historiadores usarán el cine, el video y la internet para crear un nuevo imaginario histórico que nos mostrará a todos, al fin, que la historia es una cuestión de conexión personal y emocional con lo que ha ocurrido antes. ¡Historiadores del mundo uníos! No tenéis nada que perder más que vuestras notas al pie ${ }^{1}$.

Una mañana, un profesor de una institución educativa en Bogotá propuso a sus estudiantes de grado noveno la proyección de la película Sumas y restas (2004) con la intención de enseñarles acerca de la problemática del narcotráfico que se vivió en la década de 1980 en Colombia. En ese momento, con la propuesta formativa del profesor, se encontraron en la sala de proyección las vivencias de los estudiantes, la historia, la didáctica y el cine. En esa jornada acontecieron situaciones y procesos que se relacionan con las preocupaciones teóricas, los fundamentos conceptuales, las metodologías de investigación, los discursos históricos, educativos y fílmicos que se reflexionan en este artículo.

Con la incursión de la fotografía y de las imágenes en movimiento en el espacio escolar, emergieron en la pedagogía y la didáctica reflexiones y debates sobre el sentido y el lugar que aquellas deberían ocupar en los discursos educativos. En los inicios de la producción cinematográfica, a finales del siglo XIX, se propuso que ella contribuyera a los procesos educativos de la sociedad occidental. En esa medida, es interesante advertir cómo el cine, además de la posición que adquirió en el mercado cultural como medio de esparcimiento, entretenimiento y recreación, se analizó en clave de sus sentidos educativos.

Desde la pedagogía y la didáctica de la historia se desarrollaron importantes trabajos que permitieron comprender cómo el cine se proyectó en el aparato escolar como un dispositivo que contribuyó a configurar identidades, a representar las nociones de tiempo-espacio y a cuestionar las interpretaciones del mundo. En este marco de investigaciones se asumió, como perspectiva 
metodológica, el análisis del discurso a partir de la revisión documental y de la realización de entrevistas y trabajo de campo con cinco profesores de ciencias sociales de instituciones educativas de Bogotá, de carácter público y privado de la ciudad, durante el segundo semestre del 2015 y el primer semestre de 2016. Con esta información se evidenció que los profesores en sus prácticas de enseñanza de la historia han incorporado los contenidos del tiempo presente e integran el uso del cine del narcotráfico; reconocen los lineamientos y proyectos del Ministerio de Educación Nacional y la Secretaría de Educación del Distrito que dan cuenta de las perspectivas y procesos regulados de enseñanza de la historia y apropian aspectos de la producción académica sobre las relaciones entre la formación política, la reconstrucción de la memoria social y propuestas en enseñanza de las ciencias sociales.

\section{Rejilla de análisis y condiciones de estudio}

El acontecimiento es una vibración, con una infinidad de armónicos o de submúltiplos, como una onda sonora, una onda luminosa, o incluso una parte del espacio cada vez más pequeña [...] Esta noche hay concierto. Ese es el acontecimiento ${ }^{2}$.

Pensar las experiencias de los profesores se fundamentó a partir de tres ejes analíticos: discursos, ámbitos de problematización y categorías. Estos se constituyeron en territorios de saber que se posicionan como parte de la enunciabilidad y la visibilidad del conocimiento y las prácticas de enseñanza de la historia del tiempo presente en el aparato escolar ${ }^{3}$. Con el propósito de complejizar la irrupción del cine "narco" en las prácticas de enseñanza de la historia del tiempo presente en Bogotá, se caracterizó la configuración del discurso histórico escolar a partir de la pregunta: ¿Cuáles son los rasgos y los supuestos del discurso educativo de la historia del tiempo presente que se enuncian y visibilizan en las prácticas de enseñanza de la historia escolar a partir de la circulación y uso de la cinematografía del narcotráfico como dispositivo didáctico?

Este cuestionamiento se desarrolló, por unlado, en el análisis de los discursos: histórico, educativo y fílmico; por otro, en los ámbitos de problematización que se refieren a los objetos de estudio de la historia (historia del tiempo presente y

2 Gilles Deleuze, El saber: curso sobre Foucault (Buenos Aires: Cactus, 2013).

3 Gilles Deleuze, El saber (2013), 189. 
cine del narcotráfico en Colombia), la pedagogía (sujeto escolar), y la didáctica de la historia (prácticas de enseñanza). Y, finalmente, en las categorías de análisis en las que se identificaron los enunciados que acontecen en asuntos como las tradiciones inventadas de la educación histórica (código disciplinar de la historia), las intencionalidades o propósitos de la enseñanza (horizonte formativo), las herramientas y los recursos de la labor didáctica (dispositivo didáctico) y los conceptos, ideas, nociones y concepciones del pasado-presente (conocimiento histórico escolar).

Por tanto, la perspectiva teórica que se asumió fue el análisis del discurso en las disciplinas sociales y, particularmente, en la historia; la cual se apropió de unos presupuestos teóricos y metodológicos para hacer posible otra manera de contar, escribir, problematizar y analizar las relaciones de pasado-presentefuturo. En esta perspectiva, se argumentó que la relación entre el ámbito subjetivo de los individuos y los condicionamientos objetivos de la realidad social era más compleja de lo que se había previsto, por lo que se postuló que este criterio teórico-metodológico era distinto al propuesto por la versión de la historia social ${ }^{4}$.

En síntesis, se postuló que la realidad social brinda a los sujetos las condiciones materiales de sus prácticas, pero no las valoraciones, los supuestos y los significados en las que se fundamentan. En efecto, los analistas sociales de esta corriente no aceptaron que la posición de un individuo o las manifestaciones de una problemática sea determinada o establecida por su ubicación en la estructura social. Esto propició romper con las interpretaciones y problematizaciones que daban por hecho que, ante un fenómeno social como la pobreza, la guerra o el tráfico ilícito de drogas, las respuestas que explicarían las actitudes, los significados y los comportamientos individuales y colectivos fueran en sí mismas referencias a una realidad objetiva ${ }^{5}$.

De ello resulta necesario admitir que, para esta teoría social, es el conjunto de categorías, ideas, supuestos y valores que, en un momento dado de los procesos históricos y sociales, son la base de las prácticas de los sujetos y se constituyen en la rejilla conceptual que posibilita la organización de las relaciones sociales, y se entiende que estas no son solamente el reflejo subjetivo de las características

4 Miguel Ángel Cabrera Acosta, «Historia y teoría de la sociedad. Del giro culturalista al giro lingüístico», en Lecturas de la historia. Nueve reflexiones sobre historia de la historiografía, eds. Carlos Forcadell e Ignacio Peiró, 255-72 (Zaragoza: Institución Fernando el Católico, 2002), 261. https://ifc.dpz.es/recursos/publicaciones/22/93/_ebook.pdf

5 Cabrera Acosta, 261. 
objetivas de la realidad social. Es en el lenguaje, y a partir de los discursos, que se configura, se expresa y se constituye el ámbito de los significados y las valoraciones de la realidad social.

\section{Discurso histórico escolar}

Los discursos históricos se asumieron como los conceptos, las ideas, los supuestos y las valoraciones que se configuran social y culturalmente sobre el pasado de una sociedad y sus implicaciones para el presente. En este sentido, estos discursos se construyen a partir de relaciones de poder-saber que delimitan y regulan lo que es posible que se diga sobre el pasado. Así mismo, se constituyen ideas sobre lo temporal-espacial que ubican a los individuos en el trasegar de los acontecimientos y les permiten interactuar en el conjunto de relaciones en las cuales se desempeñan. Este discurso se ocupa de construir los hechos del pasado y de dotarlos de significado.

En el caso de Bogotá, con los profesores entrevistados y a partir del trabajo de campo, se reflexionó sobre las cuestiones que dejan estas experiencias de enseñanza de la historia como elementos que sustentan unas prácticas que transforman las rutinas, los valores y las condiciones de la historia escolar. Estos enunciados de los profesores se analizaron como modos de configuración de un discurso histórico escolar que se posiciona en una dirección divergente, en lo posible, de las políticas educativas de corte eficientista, de las prácticas en las que imperaba la repetición y memorización de información, de la transposición mecánica de los contenidos que desarrollan las disciplinas sociales en una lista de temáticas de los planes de estudio escolares de las ciencias sociales, y de la homogeneización cultural del tiempo presente que imponen los medios masivos de comunicación. La pregunta que orientó el análisis fue: ¿Qué posibilidades de posicionarse como práctica de enseñanza de la historia del tiempo presente tienen los problemas sociales y el cine como fuente histórica?

En las experiencias de los profesores de ciencias sociales, se evidenció la importancia que ha adquirido el cine del narcotráfico en sus prácticas de enseñanza de la historia del tiempo presente. En efecto, la enseñanza de esta problemática social y el uso del cine en cuanto dispositivo didáctico ${ }^{6}$ se asumieron como una perspectiva de trabajo que hace realidad la historia enseñada como una posibilidad de cambio y acontecimiento pedagógico en el

6 Julieta Armella y Silvia Grinberg, «¿Hay un hipertexto en esta clase? Dispositivos pedagógicos, tecnología y subjetividad», Revista Signos y Pensamiento 31, n. ${ }^{\circ} 61$ (2012): 108-124, http://revistas.javeriana.edu.co/index.php/signoypensamiento/article/view/4412/3327. 
tiempo y en el espacio. Estas prácticas de enseñanza, entonces, no solamente se estudiaron como el deber ser obligado, sino como unas apuestas discursivas que dieron sentido a lo que se está haciendo y a las formas en que el discurso histórico escolar se reconfigura en un contexto social como el de la ciudad de Bogotá en donde las tribulaciones de los conflictos sociales se hacen latentes a cada momento.

\section{Discurso educativo}

En este caso, los enunciados y las visibilidades se refieren a la manera en que una sociedad configura los supuestos, los valores y los ideales sobre lo que debe ser la formación y la educación de sus integrantes. El discurso educativo se manifiesta sobre los espacios en que se debe educar al individuo, las formas como se deben llevar a cabo estos procesos y quiénes deben hacerlo, el valor que se le da a los conocimientos que se deberían aprender y cómo se sistematizan dichos saberes y el papel del Estado en todo ello.

En este discurso, se configuró el sujeto de la enseñanza y las teorías que explican los aprendizajes, regulado desde prácticas que definen el deber ser de los contenidos y de los métodos de enseñanza. Para esta reflexión se referenciaron tres prácticas discursivas educativas: pedagógica ${ }^{7}$, didáctica de la historia ${ }^{8}$, y pedagogía pública ${ }^{9}$. La sociedad, a través de las instituciones,

7 Oscar Saldarriaga, «Pedagogía, conocimiento y experiencia: notas arqueológicas sobre una subalternización», Revista Nómadas, 25 (2006): 98-108, http://redalyc.uaemex.mx/src/inicio/ ArtPdfRed.jsp?iCve=105115224009.

8 Raimundo Cuesta Fernández, Los deberes de la memoria en la educación (Barcelona: Ediciones Octaedro, 2007); Sebastián Plá y Joan Pagès, «Una mirada regional a la investigación en enseñanza de la historia en América Latina.», en La investigación en enseñanza de la historia en América Latina, eds. Sebastián Plá y Joan Pagès, primera edición (México: Bonilla Artigas Editores y Universidad Pedagógica Nacional, 2014), 13-39; Sebastián Plá y Joan Pagès, eds., La investigación en la enseñanza de la historia en América Latina, primera edición (México: Bonilla Artigas Editores y Universidad Pedagógica Nacional, 2014); Sandra Rodríguez Ávila, «Enseñanza y aprendizaje de la historia en Colombia. 1990-2011», en La investigación en enseñanza de la historia en América Latina, eds. Sebastián Plá y Joan Pagès, primera edición (México: Bonilla Artigas Editores y Universidad Pedagógica Nacional, 2014), 109-54; Sandra Rodríguez Ávila y Wilson Acosta Jiménez, «La emergencia de la didáctica de las ciencias sociales: ¿campo en consolidación o en disgregación?», Folios, 25 (2007): 37-52, http://www. scielo.org.co/pdf/folios/n25/n25a03.pdf.

9 Henry Giroux, Cine y entretenimiento. Elementos para una crítica política del filme (Barcelona: Paidós, 2003); Nilson Fernandes, «Educação, cinema e alteridade», Educar, 26 (2005): 67-79, http://www.scielo.br/pdf/er/n26/n26a06.pdf. 
genera estos mecanismos y las concepciones que los orientan. A la vez, se presentó como un campo en disputa por los referentes y modelos a seguir; es el caso de la escolarización. Se movilizó con el cine y el estudio de problemas sociales complejos la oportunidad de generar pensamiento y posturas críticas en las clases de historia:

El cine se constituye en una herramienta que permite analizar el tipo de sujetos que muchas veces no se trabajan en los libros de texto. Se empieza a visibilizar otras personas y otras maneras de vivir y otras maneras de sentir. Es una posibilidad en cuanto los profesores estén comprometidos, en la cuestión de leer y de hacer las relaciones entre la parte teórica y conectarlo con el mundo cultural de los estudiantes. Es importante el diálogo de saberes ${ }^{10}$.

La pregunta recurrente en los procesos de formación de profesores de historia y ciencias sociales es para qué se enseña lo que se enseña. Asunto que no es menor teniendo en cuenta que allí se definen aspectos del sentido político de los procesos formativos y estos, a su vez, inciden en la selección de los contenidos y en el cómo se van a enseñar. En el ejercicio profesional, se tiende a naturalizar la justificación que se da a los contenidos y a las metodologías de enseñanza, pues se asumen como el deber ser o como parte de una tradición. Por un lado, se exploró lo que se dice en relación con las normativas sobre la enseñanza de las ciencias sociales y la posición de los profesores al respecto; por otro lado, se identificaron otras variables sobre la pertinencia, desde el punto de vista de los profesores, para enseñar la problemática del narcotráfico, que se relacionan con el contexto de las instituciones educativas, las apuestas pedagógicas y didácticas, los intereses desde las disciplinas sociales, entre otros.

También se identificó que la pertinencia de la enseñanza de la historia del tiempo presente del narcotráfico pasó por reconocer la postura de los profesores frente a la normativa vigente de esta disciplina escolar. En este marco normativo se precisan elementos de distanciamiento o de regulación desde los cuales los maestros puedan configurar su criterio. Sin embargo, quedan abiertas las opciones de enseñar a partir de las películas las problemáticas sociales desde la perspectiva de formación y criterios políticos del profesor. En la propuesta del profesor C. Julio, están presentes estos elementos del discurso histórico

${ }^{10}$ Paola Jaime, El cine en la enseñanza de la historia, entrevistado por Wilson Acosta Jiménez, 25 de mayo de 2016. 
escolar a partir del cine que busca ir más allá del lugar ejemplarizante que se le ha concedido en la disciplina escolar:

En las clases he proyectado El muro, de Pink Floyd, que la trabajamos con unos estudiantes y la de Avatar, para mirar todo el tema de la economía extractiva en el país y los impactos que tiene sobre unos ecosistemas estratégicos. Fue con grado noveno. Los estudiantes muestran interés, frente a este tipo de cine que puede ser enmarcado como en lo que se llama la industria comercial o de Hollywood, pero desde ahí se pueden retomar cosas y se pueden retomar elementos desde los cuales se puede trabajar, como es el caso de la relación que tiene con la naturaleza e ir más allá de una relación de dominación y ver una relación más equilibrada. Para ver elementos más espirituales y ver la manera como entra la guerra ${ }^{11}$.

\section{Caracterizar las experiencias}

Toda película, no importa su duración ni su estructura, cuenta la historia del mundo, retrata al universo en su conjunto ${ }^{12}$.

En Colombia se dan hitos en los cuales resurge la preocupación por las cuestiones que se enseñan del pasado lejano y cercano en el ámbito escolar. Momentos coyunturales en los cuales sectores sociales agencian discusiones y propuestas sobre lo que debería pasar con los contenidos, los objetivos y los métodos de transmitir el pasado a las nuevas generaciones:

El miedo a abordar en la escuela de manera directa el conflicto y la violencia finalmente se convierte en un mecanismo que favorece la minimización de su trascendencia y el desdén alrededor del reconocimiento de las responsabilidades particulares y los impactos diferenciados ${ }^{13}$.

${ }^{11}$ Camilo Julio, El cine en la enseñanza de la historia, entrevistado por Wilson Acosta Jiménez, 18 de mayo de 2016.

${ }^{12}$ Gabriel Trujillo Muñoz, «Cine eres y en cine te convertirás», Revista Estudios sobre las Culturas Contemporáneas 2, n. ${ }^{\circ}$ (1996): 126, http://www.redalyc.org/pdf/316/31600407.pdf.

${ }^{13}$ María Isabel González Moreno, «La violencia y la enseñanza de la historia nacional en el escenario institucional colombiano (1948-2006)», An Institutional Colombian Perspective 8, n. $^{0} 1$ (2015): 125, http://www.scielo.edu.uy/scielo.php? script=sci_arttext\&pi$\mathrm{d}=\mathrm{S} 1688-74682015000100005 \& \operatorname{lng}=$ es\&nrm=iso. 
De ahí que, en el contexto colombiano de formación de profesores de ciencias sociales e historia de las primeras décadas del siglo XXI, se ubicó en el debate público y en la configuración de propuestas de enseñanza de la historia reciente la preocupación por asumir problemáticas sociales relevantes como el conflicto social y armado, el narcotráfico, la crisis ambiental, entre otros, en el marco de los cambios en los contenidos de la historia escolar y de los procesos de reconstrucción de las memorias colectivas ${ }^{14}$.

Por lo anterior, y como ejercicio de reflexión, se delimitaron y analizaron los ámbitos de problematización que hacen referencia a los objetos de estudio que se configuran a partir del análisis de los discursos. Estos se observaron e interpretaron como las preocupaciones de este artículo en términos teóricos, como los motivos que tienen sus implicaciones a la hora de reflexionar sobre ellos mismos y su proyección en los procesos formativos. Estos ámbitos son ejes transversales de reflexión en este escrito.

Por lo cual, se asumió que la historia del tiempo presente tiene que ver con los estudios históricos que se adelantan sobre el pasado que constituye al presente, es decir, a los procesos sociales y los acontecimientos que definen a las generaciones que los han vivido y hacen parte del relato que las identifican. La historia del tiempo presente es una preocupación de la Historia y otras disciplinas sociales por comprender los supuestos y valoraciones que en el diario acontecer se asignan a las problemáticas sociales ${ }^{15}$.

En el encuentro con los relatos de los profesores se identificaron elementos de una historia enseñada del tiempo presente que se compromete con una apuesta de formación política, a partir de sus prácticas. Esta perspectiva en la historia escolar rompe con los esquemas de formación política imperantes en el ámbito escolar, caracterizados por una concepción dogmática y restringida en la constitución del sujeto político. En la escuela se ha dado a las ciencias sociales el papel de formación de los ciudadanos en lo referente al respeto y el reconocimiento de un pasado común, unos valores compartidos, unas formas de participación e integración social. En el trabajo que realizan los profesores

${ }^{14}$ Plá y Pagès, «Una mirada regional a la investigación », 20.

${ }^{15}$ François Bédarida, «Definición, método y práctica de la historia del tiempo presente», Cuadernos de Historia Contemporánea, n. ${ }^{\circ} 20$ (1998): 19-27, https://revistas.ucm.es/index.php/ CHCO/article/download/CHCO9898110019A/7004; Hugo Fazio, «La historia del tiempo presente una historia en construcción», Revista Historia Crítica, n. ${ }^{\circ} 17$ (1998): 1-11, https:// dialnet.unirioja.es/servlet/articulo? codigo=2182434; Hugo Fazio, «La historia del tiempo presente y la modernidad mundo», Revista Historia Crítica, n. ${ }^{\circ} 34$ (2007): 184-207, https:// doi.org/www.redalyc.org/pdf/811/81103409.pdf. 
entrevistados, se evidenció la preocupación por una formación política en sus clases de historia que desestructura las ancladas visiones de la formación de sujetos dóciles y disciplinados, o dispuestos a ser buenos consumidores. La pregunta que orientó la entrevista fue: ¿Qué sentido político y formativo se podría proyectar a partir de la enseñanza de la historia reciente de los problemas sociales y el trabajo del cine como fuente?

En las respuestas se reconoce un cambio de concepción en relación con el deber ser de la historia escolar y sus implicaciones formativas para la vida de los estudiantes y las apuestas formativas de los profesores en ejercicio; cuestión que acarreó mirar en detalle estos supuestos y valores concedidos a las prácticas de enseñanza como formas de producción de un conocimiento histórico que irrumpe en las vidas de los jóvenes y las transforman. De acuerdo con los profesores, no es suficiente tener una mirada crítica sobre el problema social del narcotráfico, se necesita, además, cambiar los esquemas de los estudiantes sobre sus relaciones con el conocimiento histórico de modo que puedan explicar la complejidad de las dinámicas familiares, culturales y económicas que los atraviesan.

Es por esto que, en los discursos de los profesores de ciencias sociales, se reconoció a un sujeto escolar. En ellos y en ellas van a confluir los enunciados analizados, pues se refieren al sujeto de la enseñanza de la historia en el contexto escolar. Este sujeto se configura históricamente y actúa de acuerdo con las condiciones institucionales, así como por sus concepciones y valoraciones de su profesión ${ }^{16}$.

Lo planteado por los profesores entrevistados sorprende e invita a repensar la enseñanza, la formación de los profesores, el reconocimiento del cine en su complejidad y, en últimas, propone vivir otra historia escolar a partir de la experiencia, de los errores y de los aciertos. Se trata de construir un proyecto de historia escolar que movilice a pensar la didáctica y la pedagogía como saberes constitutivos de su ejercicio profesional, así como a apropiar los desarrollos de las disciplinas sociales para que la versión fílmica no quede reducida a una verdad incuestionable. En palabras de una profesora:

${ }^{16}$ Álvaro Acevedo Tarazona y Gabriel Samacá Alonso, «La política educativa para la enseñanza de la historia de Colombia (1948-1990): de los planes de estudio por asignaturas a la integración de las ciencias sociales», Revista Colombiana de Educación, n. ${ }^{0} 62$ (2012): 221-44, http://www.scielo.org.co/pdf/rcde/n62/n62a12.pdf; Sandra Rodríguez Ávila, «Construcción de la memoria oficial en el centenario de la Independencia: el Compendio de Historia de Colombia de Henao y Arrubla», Folios, n. ${ }^{0} 32$ (2010): 23-42, http://www.scielo.org.co/pdf/ folios/n32/n32a02.pdf. 
Se necesitan de otros saberes y ampliar los debates de la didáctica de la historia [...] En la didáctica de la historia se necesitan de otros campos de saber. Se necesita hablar con el profesor de arte, literatura y hasta con el de matemáticas. ¿Cómo construir ese conocimiento histórico desde otras disciplinas? Esto permitirá que en la didáctica de la historia se amplíen sus debates frente a como se debería hacer la transposición didáctica. ¿Cuál sería la articulación entre el conocimiento disciplinar, el conocimiento didáctico y el conocimiento pedagógico? Esto a veces no lo tenemos claro [...] se da una ruptura entre lo que uno enuncia y lo que hace en la práctica como tal. Como se da la relación de estos conocimientos con el cine [...] uno a veces estas relaciones las tomas tan naturales que no se preocupa en reflexionarlas [...] ni en cuestionarlas [...] tomamos el cine como la herramienta para que los estudiantes vayan y verifiquen todo lo que ya les he dicho atrás ${ }^{17}$.

Los aportes de los profesores entrevistados propenden a pensar la historia enseñada, no como una cuestión de utopías a realizar, sino como cuestionamientos y experiencias que obligan a pensar la enseñanza en cuanto acontecimiento. El cine en las clases de ciencias sociales ha contribuido a pensar de otro modo los contenidos, las metodologías y los fines formativos que persiguen los profesores. Con estas reflexiones, se piensan de otro modo las prácticas de enseñanza de la historia a partir del cine del narcotráfico, en el sentido de aproximarse, no a juzgar si son correctas o adecuadas estas experiencias en el contexto de la historia escolar en Bogotá, sino a caracterizarlas e identificar los enunciados que potencian apuestas por reconfigurar rasgos del código disciplinar de la historia escolar. Se "cartografió" el tipo de conocimiento histórico escolar que circula en los relatos de los profesores sobre las cuestiones que se pretende posicionar como urgentes para que las nuevas generaciones comprendan el presente que viven a diario.

En relación con las prácticas de enseñanza de la historia se pensaron con las formas en las cuales intervienen los avatares formativos de los docentes, las características de las instituciones educativas, las dinámicas culturales, los cambios generacionales y otros factores que determinan las decisiones en los modos de enseñar ${ }^{18}$.

17 Jaime, El cine en la enseñanza de la historia.

${ }^{18}$ Karen Elizabeth Catelotti y Nicolás Gabriel de Rosa, «Cine e historia en las aulas: una aproximación a la incorporación y significación del recurso en las escuelas de concepción del Uruguay», Revista Clío \& Asociados, n. ${ }^{\circ} 19$ (2014): 454-79, http://sedici.unlp.edu.ar/ handle/10915/47745; Yolanda Mercader Martínez, «El cine como espacio de enseñanza, 
En las vivencias de los profesores, la definición de contenidos se da por sus intereses académicos y políticos, de lo cual se desprende que, en la tradición sociocultural de la historia escolar, en los textos visibles como en los programas y en los manuales escolares, la decisión de los profesores sobre el qué y el cómo de la enseñanza se define por sus preocupaciones y orientaciones políticas. Cuestión que implica que el sujeto que enseña se enuncie desde el distanciamiento del deber ser, que impone los programas curriculares y los manuales escolares. El sujeto se interpreta, entonces, como un tipo de profesor que asume los lineamientos curriculares en cuanto un componente de su práctica, sin ser este el determinante de sus opciones del conocimiento histórico que circula en el ámbito escolar.

En otra entrevista, la postura del profesor va más allá y señala que la política pública no regula y es ineficiente en esta cuestión:

La política pública en educación es ineficiente hasta en controlar, digamos se da una intención un propósito de regular los contenidos a través de la estandarización y todo lo que ya se conoce, pero es demasiado deficiente el control porque los estándares pretenden una formación ciudadana y del enfoque de formar sujetos dóciles, y que participen desde las reglas de juego establecidas, que sean cuerpos disciplinados con unas capacidades mínimas para interactuar en el mercado [...] Lo cuestionable es que los docentes reproducen este tipo de control, mucho docente de ciencias sociales ha naturalizado ese control, que se da en las aulas. Pero en mi caso particular no está tan limitado ${ }^{19}$.

La importancia de la regulación es su imposición sobre los cuerpos y las conductas de los estudiantes, es un asunto que se mantiene. Pero, en el relato

producción e investigación», Reencuentro, n. ${ }^{\circ} 63$ (abril de 2012): 47-52, http://www.redalyc org/pdf/340/34023237007.pdf; Gustavo González Valencia y Carlos Valencia Calvo, «La didáctica de las ciencias sociales en Colombia: planteamientos teóricos y estado de la cuestión de la investigación.», en Una mirada al pasado y un proyecto de futuro. Investigación e innovación en didáctica de las ciencias sociales, ed. Joan Pagès y Antoni Santisteban, vol. 1, 2 vols. (Barcelona: Universitat Autònoma de Barcelona, Servei de Publicacions, Asociación Universitaria de Profesorado de Didáctica de las Ciencias Sociales, 2013), 597-605; Sandra Rodríguez Ávila, «Formación de maestros para el presente: memoria y enseñanza de la historia reciente», Revista Colombiana de Educación, n. ${ }^{\circ} 62$ (junio de 2012): 287-314, http:// revistas.pedagogica.edu.co/index.php/RCE/article/view/1640/1592.

${ }^{19}$ Henry Gómez, El cine en la enseñanza de la historia, entrevistado por Wilson Acosta Jiménez, 16 de mayo de 2016. 
del profesor, se evidencia que, en términos de los contenidos y del cómo se desarrollan en el aula, se pueden explorar distintas opciones.

Y en cuanto, al ámbito del cine del narcotráfico en Colombia se problematizó la visión de conjunto, la forma como en estas cinematografías se han abordado la cuestión del tráfico ilegal de drogas en cuanto asunto que implica a los individuos y que contribuye a pensar las relaciones sociales, los conflictos políticos, los intereses económicos y otras dinámicas que afectan la vida cotidiana $^{20}$.

De acuerdo con los profesores, con la irrupción de los filmes se cuestiona y se desplaza la hegemonía de los manuales escolares, pues estos se imponían como parte de una directriz institucional, la cual no se puede dejar de lado, entre otros motivos, por los costos para los padres de familia del texto escolar y por la información que este ofrece para labor didáctica. Con respecto a la dispersión en los contenidos y fines de la enseñanza de la historia, con la proyección de películas los textos escolares dejan de ser el centro de información o desde el cual se definen las temáticas a desarrollar. El texto escolar se asumió como un componente que contribuye a cuestionar lo que se muestra en una película o en los relatos de unos hechos históricos como los que se describen en la obra literaria La franja amarilla de William Ospina ${ }^{21}$. En últimas, se encuentra en los relatos de los profesores un distanciamiento de la regulación en los contenidos y metodologías que representan los textos escolares en la enseñanza de la historia escolar.

En definitiva, el acontecimiento del cine del narcotráfico en las prácticas de enseñanza emerge de forma silenciosa en las fisuras que quedan de los rasgos fuertes del código disciplinar, como la rigidez en las temáticas y actividades que caracterizaban a la historia regulada que se visibiliza en los textos escolares.

En el ya citado estudio de Johnsen hay un amplio capítulo dedicado a esta cuestión de la que ahora solo nos interesa destacar que, de forma bastante habitual, el que alguna cuestión esté presente en los manuales no garantiza en absoluto que esta sea tratada en el aula o, lo que es prácticamente lo mismo, que lo que enseñan los profesores suele estar en los libros, pero que estos no enseñan todo lo que está en los mismos ${ }^{22}$.

\footnotetext{
${ }^{20}$ Suárez, «Cinembargo Colombia: ensayos críticos»..

${ }^{21}$ William Ospina, La franja amarilla (Bogotá: Norma, 1999).

${ }^{22}$ Rafael Valls Montés, «Los estudios sobre los manuales escolares de historia y sus nuevas perspectivas», Revista Didáctica de las Ciencias Experimentales y Sociales, n. ${ }^{\circ} 15$ (2001): 25.
} 


\section{Horizonte formativo}

El problema social del narcotráfico es un contenido de la historia escolar que se incluyó en los planes de estudio y los proyectos pedagógicos de la enseñanza de las ciencias sociales. Esta temática se aborda en los grados de 9.., $10 . \stackrel{\circ}{y}$ 11.ำ de la educación básica y media; los profesores señalan que hace parte de la reflexión sobre los problemas de la historia reciente de los colombianos. Ahora bien, esta temática se enseña, por lo general, a partir de la proyección de películas que muestran aspectos de la producción, tráfico y consumo de sustancias prohibidas. Los profesores entrevistados referenciaron cuáles fueron los propósitos formativos que se propusieron a la hora de abordar la problemática social del narcotráfico con la presentación de películas como Sumas y restas (2004), El rey (2004), Rodrigo D. Nofuturo (1999), y Rosario Tijeras (2005), entre otras. En la entrevista del profesor Cabrera, A., se señaló como:

Los procesos de enseñanza de la historia reciente se enunciaron básicamente desde la formación política con una intencionalidad de formar el pensamiento crítico. Este pensamiento no supera la lógica de diagnóstico y entonces este si le permite ver a los estudiantes y docentes ver las dinámicas de estructuración las lógicas de desigualdad social, pero lo que no tiene es una posibilidad de tipo proyectivo ${ }^{23}$.

En efecto, la historia del tiempo presente del narcotráfico se enmarca en un horizonte de sentido que se proyecta como el cuestionamiento a las realidades vividas por los sujetos escolares con la posibilidad de afectar las miradas que se naturalizaron culturalmente y el papel de los medios de comunicación en los procesos de enseñanza ${ }^{24}$. En las condiciones históricas que se dan en Colombia de conflictos sociales, esto implica que la comprensión de las relaciones entre el pasado y el presente se asuma como una labor compleja del análisis de lo que somos como sociedad. Esto propicia que, en medio de la diversidad de comunidades y de perspectivas de vida, es necesario pensar las cuestiones que obligan a tomar una posición política y ética. En el discurso de los profesores se reconoce la pertinencia de la enseñanza de los contenidos de la historia

${ }^{23}$ Cabrera, El cine en la enseñanza de la historia.

${ }^{24}$ Miguel López Rodríguez y Laura Arias Ferrer, «Los medios audiovisuales para la enseñanza de la historia del mundo contemporáneo: la guerra fría», en Medios de comunicación y pensamiento crítico. Nuevas formas de interacción social, eds. Juan José Díaz Matarranz, Antoni Santisteban y Áurea Cascajero Garcés (Alcalá de Henares: Universidad de Alcalá, 2013), 479-490, http:// didactica-ciencias-sociales.org/wp-content/uploads/2013/11/publiGDJA.pdf. 
del tiempo presente, por ser necesaria para explicar el contexto mismo de los estudiantes y de los docentes.

Es decir que, en otras circunstancias, lo prioritario fue la enseñanza de temáticas que estuvieran vinculadas con épocas y lugares lejanos que contribuyeron a formar interpretaciones del pasado como un asunto alejado del presente y, a la vez, que sirviera de justificación del orden social. En las condiciones del presente, argumentan los profesores entrevistados, es la misma dinámica vertiginosa de los procesos políticos, sociales y económicos del territorio colombiano como la vulneración de derechos humanos, el conflicto armado y social, la crisis ambiental, entre otros, que posiciona el carácter urgente y necesario de generar procesos formativos que aporten a los estudiantes a comprenderlos y a emprender opciones de transformación.

Lo que pasa es que la escuela sí adolece de una conciencia histórica fuerte, es lo que sucede, los problemas sociales y la historia reciente solamente le debe importar al profesor de ciencias sociales, entonces una de las luchas de los docentes del área es que esto se trabaje no de manera transversal, sino que se generen espacios en donde todos como docentes de todas las áreas y con los estudiantes participen activamente en la reconstrucción de la memoria. Más ahora con el proceso de paz, y se van logrando cosas [...] este año que vamos a hacer un foro y una fiesta por la paz y la memoria, para finales de octubre donde los mismos docentes den su visión de la historia y del conflicto [...] hacer una reconstrucción entre todos de que es lo que ha venido pasando [...] y porque a todos nos ha afectado ${ }^{25}$.

El trabajo didáctico y pedagógico con los filmes en el aula cuestiona los valores, los conocimientos, los sentimientos y los deseos que se asumen como dados por la experiencia de los sujetos escolares y por las imágenes que saturan la cotidianidad. Las películas en su acontecer del para qué de la historia escolar se constituyen en un desbordamiento al estado de "naturalidad" con el que se asumen las informaciones de los medios de comunicación como los noticieros y las vivencias habituales. No obstante, este horizonte formativo es confrontado por las condiciones de las instituciones educativas, en las que se dieron situaciones como la siguiente:

En el contexto del colegio abordar estas problemáticas, pues recuerdo como una vez nos dijeron que esos temas eran apología a la guerrilla, al crimen...

${ }^{25}$ Gómez, El cine en la enseñanza de la historia. 
que la historia no debería tocar eso, según unos padres de familia... como silenciar esas voces ${ }^{26}$.

En el relato de Gómez, H., se resalta la actitud del padre de familia y de la estudiante que explicitan su inconformidad por el estudio de los hechos y los actores sociales que se vinculan al conflicto armado. En los medios de comunicación social se tiende a utilizar el mecanismo de presentar informaciones que apologizan a determinados actores del conflicto para mostrar sus virtudes o sus vicios y posicionar miradas maniqueas. El sentido formativo en el ámbito escolar pasaría por desestructurar estas lecturas y miradas dualistas entre los "buenos" y los "malos". Así, los contenidos que se proyectan en la historia escolar a partir del cine no se asumen como un reflejo o una representación dada de las vidas, de los conflictos, de los sueños y de las esperanzas. Con lo cual resulta necesario admitir que los contenidos de la historia del tiempo presente adquieren sentido en la medida que se tienen en cuenta las vivencias de los sujetos escolares y su vínculo con los problemas sociales que se enseñan.

Lo importante es generar otro tipo de pensamiento, en donde es necesario construir la esperanza, que en el día a día está la posibilidad. Mostraba el documental de Riochiquito, para vieran todo el proceso de la formación de las guerrillas y ver las raíces históricas del conflicto social2 ${ }^{27}$.

En las experiencias de los profesores Gómez, H. y Julio, C., se identifican las potencialidades del diálogo de voces (académica, vida cotidiana y cine) y de cómo este es una opción de cambio en el horizonte formativo de los contenidos en la enseñanza de la historia. De hecho, en otra experiencia se relata cómo esta perspectiva formativa se reafirma:

Tenemos el caso de una niña que es particular, por lo que ella expresa de su contexto familiar se lograría deducir que la familia de ella podría tener vínculos con el narcotráfico. En las discusiones que se dan en clase ella resalta lo positivo del narcotráfico, porque dice que permite salir adelante a la gente, los narcos ayudan a las personas que el Estado ha olvidado. Ella viene de Risaralda y por sus discursos se evidencia que ha estado frente a la problemática del narcotráfico. Tenemos también el caso de una mexicana

\footnotetext{
${ }^{26}$ Gómez, El cine en la enseñanza de la historia.

${ }^{27}$ Julio, El cine en la enseñanza de la historia.
} 


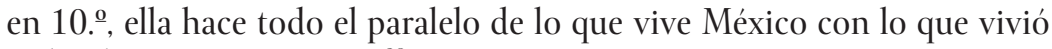
Colombia en ese momento ${ }^{28}$.

La intención formativa de la profesora es la de conectar el conocimiento escolar con la vida de las estudiantes, lo cual implicó que es un horizonte de sentido en el extrañamiento de la verdad instituida, la "desaulización” de los contenidos, la problematización del presente y la construcción de imágenes que vayan más allá de lo evidente ${ }^{29}$. La tarea de enseñar los contenidos de la historia del tiempo presente no se reduce a la transmisión de un listado de temas o al desarrollo de competencias para la obtención de resultados exitosos en las pruebas estatales y, en últimas, no se quedaría en la intención de abordar temáticas de carácter rutinario e instrumental. La enseñanza de estos problemas sociales y en esta perspectiva de formación política se asume como un trabajo a contracorriente de los cánones establecidos y en una dinámica permanente por inventar la valoración y el significado de historia escolar ${ }^{30}$.

\section{Rupturas en las prácticas de enseñanza}

En este apartado se analizó lo que dicen los profesores sobre los contenidos que se estudian de la historia del tiempo presente y su relación con los asuntos que muestra el cine del narcotráfico. Adicionalmente, se identificaron las rupturas con los rasgos que han predominado en la selección de contenidos en la enseñanza de las ciencias sociales en el ámbito escolar ${ }^{31}$. Para dar cuenta de estos cambios es importante señalar que según los profesores entrevistados los contenidos responden a propuestas curriculares diseñadas por ellos mismos, haciendo uso de su autonomía en la enseñanza, en unos casos, y en otros negociando con los lineamientos y estándares curriculares que propone el Ministerio de Educación Nacional. (En adelante Men).

28 Julio, El cine en la enseñanza de la historia.

${ }^{29}$ Cuesta Fernández, Los deberes de la memoria, 100.

${ }^{30}$ Rodríguez Ávila, «Formación de maestros para el presente ».

${ }^{31}$ Absalón Jiménez, Raúl Infante y Amanda Cortés, «Escuela, memoria y conflicto en Colombia. Un ejercicio del estado del arte de la temática», Revista Colombiana de Educación, n. ${ }^{0} 62$ (julio de 2012): 287-314, http://revistas.pedagogica.edu.co/index.php/RCE/article/ view/1640/1592; Rodríguez Ávila, «Formación de maestros para el presente»; Rocío Londoño, Mario Aguirre e Indira Sierra, La enseñanza de la historia en el ámbito escolar bogotano (Bogotá: Secretaría de Educación del Distrito - Alcaldía Mayor de Bogotá, 2015). 
En efecto, se registró el caso de la profesora Jaime, P., que relata cómo se resuelve esta cuestión en la institución educativa donde labora:

Lo que hemos discutido en el área es definir qué es lo mínimo que necesita un niño para aprobar el curso. Los estándares es la base con lo que uno trabaja, y los lineamientos, entonces miramos que están enfocados a las relaciones entre los ejes temporal - espacial, el ético político, y el eje de las organizaciones económicas y el espacio geográfico. Se ha buscado que se dé articulación entre las áreas y se adecuen a los lineamientos y los estándares ${ }^{32}$.

Esta postura deja abiertas las posibilidades para negociar con las exigencias que se imponen desde el $\mathrm{Men}^{33}$ y que, en el caso colombiano, se miden a través de las evaluaciones estandarizadas que se realizan a los estudiantes. Es importante la apuesta del equipo de profesores de esta experiencia por resolver una cuestión que no se puede convertir en el centro de labor pedagógica, es decir, convertir sus clases en un ejercicio de preparación para las evaluaciones y los estándares que exige el Men. En el siguiente relato del profesor Gómez, H., se presentan varios elementos que son objeto de análisis en relación con los contenidos de la enseñanza del narcotráfico a partir de las películas:

Hemos trabajado el Rey, Sumas y Restas y Retrato en un mar de mentiras... estas tres películas sobre la historia reciente y la Estrategia del caracol, que hace rato no se proyectan... Se trabaja sobre todo en relación con los años ochenta, y de uno que milita en la izquierda, siempre trata de mostrar el conflicto, pero en general en blanco y negro, es decir, entre una insurgencia y un Estado ${ }^{34}$.

En este relato el problema social del narcotráfico hace parte de una perspectiva de abordar los problemas sociales en la enseñanza de la historia del tiempo presente ${ }^{35}$. Se señala la importancia de abordar unos fundamentos conceptuales que, en esta ocasión, se desarrollan en el área de ciencias políticas

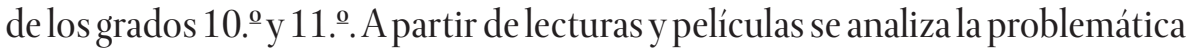
del narcotráfico. Es un problema que, como contenido de la historia escolar,

\footnotetext{
${ }^{32}$ Jaime, El cine en la enseñanza de la historia.

${ }^{33}$ Ministerio de Educación Nacional, Lineamientos curriculares para el área de Ciencias Sociales (Bogotá: Editorial Delfín Ltda, 2002).

${ }^{34}$ Gómez, El cine en la enseñanza de la historia.

${ }^{35}$ González Moreno, «La violencia y la enseñanza de la historia».
} 
en el contexto donde se desarrolla el estudio de esta problemática, se relaciona con la vida de los jóvenes estudiantes y con las condiciones en las cuales se vive en los barrios periféricos de la ciudad de Bogotá. Estos profesores de ciencias sociales y de historia se posicionan como sujetos mediadores de las culturas, del conocimiento disciplinar y las prácticas pedagógicas en la articulación de la enseñanza de problemas del tiempo presente de Latinoamérica desde la proyección de películas.

El enunciado que circula en los profesores es que a partir de los contenidos que desarrolla una película es posible que sean analizados de forma integral y romper con las lecturas y miradas segmentadas y fragmentadas de temas.

Con la película "El Arriero" (2009) fue una cuestión más de la cultura política colombiana, disoluta y traqueta, que digamos a partir de los años ochenta hace parte del conflicto colombiano. Retomamos la historia de Colombia del siglo XX, las guerras bipartidistas, el surgimiento de las guerrillas, el surgimiento de los paramilitares, de los narcotraficantes. Esto se aborda especialmente en grado noveno y décimo ${ }^{36}$.

Cuando se profundiza en el estudio de los acontecimientos que se constituyen en referentes de los momentos y espacios que han marcado a unas generaciones en particular, se pretende posicionar un discurso histórico escolar que se extraña de sí mismo, del devenir y de las formas naturalizadas de contar e interpretar lo que somos, a través de las imágenes y de la configuración de memorias sociales. La configuración de la investigación y la enseñanza del tiempo presente en la historia académica y la escolar está sujeta a una época que es saturada por imágenes de recuerdos inmediatos y lejanos que educan sobre los tiempos y espacios a las generaciones en las versiones de los acontecimientos y procesos sociales desde el presente ${ }^{37}$.

\section{Conocimientos históricos escolares}

La producción de conocimientos históricos en el aparato escolar, la visión romántica del pasado que busca generar afecto, pertenencia e identidad con el colectivo social y la visión ilustrada, que en lo fundamental pretende que el individuo explique desde la disciplina histórica los procesos sociales, entran en

36 Jenniffer Cruz, El cine en la enseñanza de la historia, entrevistada por Wilson Acosta Jiménez, 19 de mayo de 2016.

${ }^{37}$ Claudia Feld y Jessica Stites Mor, eds., El pasado que miramos. Memoria e imagen ante la historia reciente (Buenos Aires: Paidós, 2009). 
tensión con la apuesta del cine del narcotráfico en las prácticas de enseñanza; no obstante, es una tensión que se pretende superar ${ }^{38}$. El conocimiento histórico escolar a partir de las imágenes audiovisuales del tráfico de drogas se ve implicado y transformado.

Es un conocimiento que apuesta por integrar elementos que expliquen y complejicen-deconstruyan las miradas de la problemática social. Para lo cual se requiere formar la mirada en relación con lo que muestra la película, con las situaciones que se viven a diario y con la producción académica. Las películas visibilizan un conocimiento particular sobre el problema del narcotráfico, pues acercan a las vivencias, a las situaciones, a las condiciones, a los personajes que, en determinado momento, optaron por asumir la vida de narcotraficantes.

El conocimiento histórico escolar es el resultado de establecer relaciones entre la historia académica como el manejo de conceptos y métodos con la información pública y mediática sobre los procesos sociales y la formación de una postura sobre el devenir histórico. Es un conocimiento que implica información, referentes conceptuales-metodológicos, posicionamiento político y sentido de las dinámicas que afectan al individuo y al colectivo. Esta aparente claridad que se tenía en tiempos no tan lejanos sobre el sentido que se le debería dar a la formación de los individuos, hoy se encuentra puesta en cuestión y hace parte de la preocupación de los profesores a la hora de reflexionar sobre lo que acontece con sus propuestas de enseñanza de la historia del tiempo presente a partir del cine.

Por ende, es posible identificar la postura de los profesores entrevistados sobre el sentido del conocimiento que acontece en sus propuestas de enseñanza de la historia con el uso de películas como un dispositivo didáctico. En tal caso, se asume como un conocimiento situado que interactúa con la producción académica, las explicaciones cotidianas de los medios y los referentes de las vivencias personales. Lo cual implica que los profesores reconocen la importancia del cine como medio para la producción de conocimientos, así como una mirada que acude a las fuentes disciplinares y a la vida cotidiana para desentrañar-complejizar las lecturas y miradas del narcotráfico.

La enseñanza de la historia que pasa por la imagen fílmica tendría como objetivo la generación de conocimientos que provocan preguntas, que cuestionan la existencia, que se distancian de los lugares comunes sobre los

${ }^{38}$ Mario Carretero, Documentos de identidad.La construcción de la memoria histórica en un mundo global (Buenos Aires: Paidós, 2007). 
procesos sociales y que, en últimas, contribuyen a la formación de una lectura crítica del orden social y de las problemáticas sociales.

De allí que el conocimiento histórico escolar se define desde otros referentes y se posicione de otras maneras. Por esto, en primer lugar, están los elementos descriptivos del problema del narcotráfico que se presentan en la película. Este es un tipo de conocimiento que se busca que los estudiantes reconozcan al ver las películas. Por ejemplo, al ver Sumas y restas, la profesora Jaime, P. resalta como sus estudiantes describen el lugar, el momento en el que ocurrieron los hechos, los personajes y las implicaciones del tráfico de drogas en la ciudad de Medellín en la década de 1980. Esta información se constituye en la base del proceso de reconocimiento de esta problemática. Los profesores entrevistados señalan la importancia de contar con informaciones, datos y el contexto de las situaciones que se relatan en las películas.

En segundo lugar, es un conocimiento analítico en el cual se busca comprender las relaciones sociales y los procesos que el narcotráfico ha generado en la sociedad. En este punto se precisa la importancia de formar en lo conceptual y no quedarse solamente en lo narrativo; también se insiste en establecer relaciones con las disciplinas sociales. En tercer lugar, es un conocimiento reflexivo, es decir, se reconocen como sujetos que observan la película. Acontece un conocimiento que, si bien guarda relación con la tradición de la cultural letrada - ilustrada, se traslada a la interacción con una cultura oral - icónica. En los profesores se mantiene un interés por lograr en los estudiantes conocimientos elaborados sobre el pasado reciente del narcotráfico; de allí la importancia de asumir posturas teóricas, conceptos y metodologías que desde las disciplinas sociales posibiliten explicar el problema social del narcotráfico. Sin embargo, el cine abre otras posibilidades de diálogo y de confrontación con los supuestos que tienen los estudiantes sobre esta realidad.

Es en esta perspectiva que el cine se posiciona como un dispositivo didáctico que se constituye en una opción de formación de conocimientos críticos. En tal sentido, otro profesor propone que además de ver películas y analizarlas en relación con las problemáticas sociales que se estudian en las clases de ciencias sociales e historia, es necesario dar el paso a la producción audiovisual y su articulación con procesos comunitarios. No es solamente un ejercicio de mirar, sino de aprender a construir la mirada. La siguiente experiencia puede servir como ejemplo: 
Y también debemos potenciar la producción audiovisual, nosotros con el colectivo que trabajamos que se llama Madre tierra, en Usme, empezamos con un cine club y ahora estamos haciendo producción audiovisual. Porque proyectamos esas películas y otro cine club de otro colectivo también la proyecta, porque se vuelven de culto y ya nadie va porque todos la han proyectado. Entonces dijimos debemos es hacer producciones propias y $\operatorname{proyectar}^{39}$.

La relación entre pasado-presente-futuro se configura como una cuestión que históricamente permite acercarse a lectura compleja de la categoría tiempo. En los supuestos del pasado-presente de la vida diaria y el conocimiento histórico escolar, está latente una discusión sobre el concepto de tiempo y la manera cómo se define lo que somos. Es la oportunidad de cuestionar las visiones que asumen la categoría de tiempo como una cuestión lineal y naturalizada de la vida en sociedad. En este sentido, se problematiza la perspectiva de lo temporal que se posicionó en el discurso histórico escolar, en la cual predominaba la concepción que aseguraba un avance, un desarrollo y un progreso hacia una sociedad mejor.

\section{Conclusiones}

En nuestra inevitable subordinación al pasado, condenados, como lo estamos, a conocerlo únicamente por sus huellas, por lo menos, hemos conseguido saber mucho más acerca de él que lo que tuvo a bien dejarnos dicho ${ }^{40}$.

Los profesores manifestaron la necesidad de complejizar las relaciones entre cine, historia del tiempo presente, sujetos e historia escolares. Estos vínculos se evidenciaron en la configuración del conocimiento histórico escolar como la proyección en las prácticas de enseñanza de la historia de un acontecimiento que irrumpe y genera otras concepciones sobre el horizonte de sentido en lo que implica, para las nuevas generaciones, las referencias al pasado cercano y al que viven a diario. Este pasado, que hasta hace unas décadas no era objeto de la investigación de la disciplina histórica y mucho menos de la enseñanza de la historia escolar, se constituyó en un referente para posicionar sujetos que se piensan a sí mismos y a la cultura en la que habitan.

${ }^{40}$ Carlo Ginzburg, El hilo y las huellas. Lo verdadero, lo falso, lo ficticio (Buenos Aires: Fondo de Cultura Económica, 2010), 13. 
En las respuestas se reconoce un cambio de concepción en relación con el deber ser de la historia escolar y sus implicaciones formativas para la vida de los estudiantes y las apuestas formativas de los profesores en ejercicio; cuestión que acarreó mirar en detalle estos supuestos y valores concedidos a las prácticas de enseñanza como formas de producción de un conocimiento histórico que irrumpe en las vidas de los jóvenes y las transforma.

En este artículo se reflexionaron las prácticas de enseñanza de la historia escolar desde lo que dicen los profesores que acontece cuando se proyecta una película en sus actividades didácticas y, en especial, al estudiar la problemática del narcotráfico. Los profesores señalaron las rupturas con las cuestiones que tradicionalmente se enseñaban de historia y lo que esto implicó en su ejercicio profesional. Para el abordaje de estas apuestas, se establecieron cuatro categorías: código disciplinar de la historia, dispositivo didáctico, conocimiento histórico escolar y horizonte formativo. Desde estas categorías, se identificaron puntos de ruptura con las cuestiones que históricamente han caracterizado el código disciplinar de la Historia en Colombia y las implicaciones que tiene la irrupción del cine como un dispositivo didáctico. Se cuestionaron estos asuntos a partir de las siguientes preguntas: ¿Qué se dice sobre lo que acontece en las prácticas de enseñanza de la historia en relación con las rupturas y tensiones que genera la irrupción del cine como dispositivo didáctico? ¿Qué implica enseñar la problemática del narcotráfico a partir del cine con jóvenes de 14 a 16 años, en contextos sociales de sectores donde esta problemática irrumpe a cada momento o se tiene una experiencia que ha sido mediatiza por los relatos televisivos?

La indagación por las prácticas de enseñanza de la historia fue un acercamiento a lo que acontece con los profesores, los estudiantes y las expresiones que emergen de las experiencias cotidianas y complejas de los procesos formativos. Por esto, se accedió a los registros escolares de las actividades didácticas y los testimonios de los profesores que señalan los supuestos que se posicionan a la hora de abordar problemáticas recientes, como la del narcotráfico, a partir del uso didáctico de películas.

Efectivamente, se caracterizó lo que se dice y cómo se dice sobre la importancia y el sentido de abordar estas problemáticas a partir de la mirada crítica del cine. Se problematizaron las formas en que el cine termina producido en el ámbito escolar, se cuestionaron las apropiaciones didácticas y se reflexionaron las dinámicas de mediación discursiva que cumplieron las propuestas fílmicas en el escenario escolar. En estas prácticas, se identificaron 
las fisuras, las tensiones, las reiteraciones y los conflictos que se dan a la hora de colocar en diálogo dos dispositivos como el escolar y el cinematográfico, en las cuales cada uno ha entendido lo que significa educar la mirada, transmitir los valores sociales y formar en subjetividades.

\section{Referencias bibliográficas}

\section{Fuentes secundarias}

Acevedo Tarazona, Álvaro y Gabriel Samacá Alonso. «La política educativa para la enseñanza de la historia de Colombia (1948-1990): de los planes de estudio por asignaturas a la integración de las ciencias sociales». Revista Colombiana de Educación, n. ${ }^{\circ} 62$ (2012):221-44, http://www.scielo.org.co/pdf/rcde/n62/n62a12.pdf.

Armella, Julieta y Silvia Grinberg. « ¿Hay un hipertexto en esta clase? Dispositivos pedagógicos, tecnología y subjetividad». Revista Signos y Pensamiento 31, n. ${ }^{\circ} 61$ (2012): 108-24. http://revistas.javeriana.edu.co/index.php/signoypensamiento/ article/view/4412/3327.

Bédarida, François. «Definición, método y práctica de la historia del tiempo presente». Cuadernos de Historia Contemporánea, n. ${ }^{\circ} 20$ (1998): 19-27, https://revistas. ucm.es/index.php/CHCO/article/download/CHCO9898110019A/7004.

Cabrera Acosta, Miguel Ángel. «Historia y teoría de la sociedad. Del giro culturalista al giro lingüístico» En Lecturas de la historia. Nueve reflexiones sobre historia de la historiografía, editado por Carlos Forcadell y Ignacio Peiró, 255-72. Zaragoza: Institución Fernando el Católico, 2002, https://ifc.dpz.es/recursos/publicaciones/22/93/_ebook.pdf.

Cabrera, Antonio. El cine en la enseñanza de la historia. Entrevistado por Wilson Acosta Jiménez, 13 de mayo de 2016.

Carretero, Mario. Documentos de identidad. La construcción de la memoria histórica en un mundo global. Buenos Aires: Paidós, 2007.

Catelotti, Karen Elizabeth y Nicolás Gabriel de Rosa. «Cine e historia en las aulas: una aproximación a la incorporación y significación del recurso en las escuelas de concepción del Uruguay». Revista Clío \& Asociados, n. ${ }^{\circ} 19$ (2014): 454-79, http:// sedici.unlp.edu.ar/handle/10915/47745.

Cruz, Jenniffer. El cine en la enseñanza de la historia. Entrevistada por Wilson Acosta Jiménez, 19 de mayo de 2016.

Cuesta Fernández, Raimundo. Los deberes de la memoria en la educación. Barcelona: Ediciones Octaedro, 2007.

Deleuze, Gilles. El saber: curso sobre Foucault. Buenos Aires: Cactus, 2013.

Fazio, Hugo. «La historia del tiempo presente una historia en construcción». Revista Historia Crítica, n. ${ }^{\circ} 17$ (1998): 1-11, https://dialnet.unirioja.es/servlet/articulo?codigo $=2182434$. 
Fazio, Hugo. «La historia del tiempo presente y la modernidad mundo». Revista Historia Crítica, n. ${ }^{\circ} 34$ (2007): 184-207, https://doi.org/www.redalyc.org/ pdf/811/81103409.pdf.

Feld, Claudia y Jessica Stites Mor, eds. El pasado que miramos. Memoria e imagen ante la historia reciente. Buenos Aires: Paidós, 2009.

Fernandes, Nilson. «Educação, cinema e alteridade». Educar 26 (2005): 67-79, http:// www.scielo.br/pdf/er/n26/n26a06.pdf.

Ginzburg, Carlo. El hilo y las huellas. Lo verdadero, lo falso, lo ficticio. Buenos Aires: Fondo de Cultura Económica, 2010.

Giroux, Henry. Cine y entretenimiento. Elementos para una crítica política del filme. Barcelona: Paidós, 2003.

Gómez, Henry. El cine en la enseñanza de la historia. Entrevistado por Wilson Acosta Jiménez, 16 de mayo de 2016.

González Moreno, María Isabel. «La violencia y la enseñanza de la historia nacional en el escenario institucional colombiano (1948-2006)». An Institutional Colombian Perspective 8, n. ${ }^{\circ} 1$ (2015): 123-51, http://www.scielo.edu.uy/scielo.php? script=sci_arttext\&pid=S1688-74682015000100005\&lng=es\&nrm=iso.

González Valencia, Gustavo y Carlos Valencia Calvo. «La didáctica de las ciencias sociales en Colombia: planteamientos teóricos y estado de la cuestión de la investigación» En Una mirada al pasado y un proyecto de futuro. Investigación e innovación en didáctica de las ciencias sociales, editado por Joan Pagès y Antoni Santisteban, 1:597-605. Barcelona: Universitat Autònoma de Barcelona, Servei de Publicacions, Asociación Universitaria de Profesorado de Didáctica de las Ciencias Sociales, 2013.

Jaime, Paola. El cine en la enseñanza de la historia. Entrevistada por Wilson Acosta Jiménez, 25 de mayo de 2016.

Jiménez, Abasalón, Raul Infante y Amanda Cortés. «Escuela, memoria y conflicto en Colombia. Un ejercicio del estado del arte de la temática». Revista Colombiana de Educación, n. ${ }^{\circ} 62$ (julio de 2012): 287-314, http://revistas.pedagogica.edu.co/ index.php/RCE/article/view/1640/1592.

Julio, Camilo. El cine en la enseñanza de la historia. Entrevistado por Wilson Acosta Jiménez, 18 de mayo de 2016.

Londoño, Rocío, Mario Aguirre e Indira Sierra. La enseñanza de la historia en el ámbito escolar bogotano. Bogotá: Secretaría de Educación del Distrito - Alcadía Mayor de Bogotá, 2015.

López Rodríguez, Miguel y Laura Arias Ferrer. «Los medios audiovisuales para la enseñanza de la historia del mundo contemporáneo: la guerra fría». En Medios de comunicación y pensamiento crítico. Nuevas formas de interacción social, editado por Juan José Díaz Matarranz, Antoni Santisteban y Aurea Cascajero Garcés, 479-90. Alcalá de Henares: Universidad de Alcalá, 2013, http://didactica-ciencias-sociales. org/wp-content/uploads/2013/11/publiGDJA.pdf. 
Mercader Martínez, Yolanda. «El cine como espacio de enseñanza, producción e investigación». Reencuentro, n. ${ }^{\circ} 63$ (abril de 2012): 47-52, http://www.redalyc. org/pdf/340/34023237007.pdf.

Ministerio de Educación Nacional. Lineamientos curriculares para el área de Ciencias Sociales. Bogotá: Editorial Delfín Ltda, 2002.

Ospina, William. La franja amarilla. Bogotá: Norma, 1999.

Plá, Sebastián y Joan Pagès, eds. La investigación en la enseñanza de la historia en América Latina, primera edición. México: Bonilla Artigas Editores y Universidad Pedagógica Nacional, 2014.

Pla, Sebastian y Joan Pages, eds. « Una mirada regional a la investigación en enseñanza de la historia en América Latina ». En La investigación en enseñanza de la historia en América Latina, editado por Sebastián Plá y Joan Pagès, primera edición, 13-39. México: Bonilla Artigas Editores y Universidad Pedagógica Nacional, 2014.

Rodríguez Ávila, Sandra. «Construcción de la memoria oficial en el centenario de la Independencia: el Compendio de Historia de Colombia de Henao y Arrubla». Folios, n. ${ }^{\circ} 32$ (2010): 23-42, http://www.scielo.org.co/pdf/folios/n32/n32a02.pdf.

Rodríguez Ávila, Sandra. «Enseñanza y aprendizaje de la historia en Colombia. 19902011». En La investigación en enseñanza de la historia en América Latina, editado por Sebastián Plá y Joan Pagès, primera edición, 109-54. México: Bonilla Artigas Editores y Universidad Pedagógica Nacional, 2014.

Rodríguez Ávila, Sandra. «Formación de maestros para el presente: memoria y enseñanza de la historia reciente». Revista Colombiana de Educación, n. ${ }^{\circ} 62$ (junio de 2012): 287-314, http://revistas.pedagogica.edu.co/index.php/RCE/article/ view/1640/1592.

Rodríguez Ávila, Sandra y Wilson Acosta Jiménez. «La emergencia de la didáctica de las ciencias sociales: ¿campo en consolidación o en disgregación?» Folios 25 (2007): 37-52, http://www.scielo.org.co/pdf/folios/n25/n25a03.pdf.

Rosenstone, Robert. Cine y visualidad: historización de la imagen contemporánea. Santiago de Chile: Ediciones Universidad Finis Terrae., 2013.

Saldarriaga, Oscar. «Pedagogía, conocimiento y experiencia: notas arqueológicas sobre una subalternización». Revista Nómadas 25 (2006): 98-108, http://redalyc. uaemex.mx/src/inicio/ArtPdfRed.jsp?iCve=105115224009.

Suárez, Juana. Cinembargo Colombia: ensayos críticos sobre cine y cultura. Bogotá: Editorial Universidad del Valle, 2009.

Trujillo Muñoz, Gabriel. «Cine eres y en cine te convertirás». Revista Estudios sobre las Culturas Contemporáneas 2, n. ${ }^{\circ} 4$ (1996): 125-36, http://www.redalyc.org/ pdf/316/31600407.pdf.

Valls Montés, Rafael. «Los estudios sobre los manuales escolares de historia y sus nuevas perspectivas». Revista Didáctica de las Ciencias Experimentales y Sociales, n. ${ }^{\circ} 15$ (2001): 23-36. 


\section{Filmografía}

Calle, G. (Director) (2009). El arriero [Película]. Colombia: Coproducción Colombia-España; Fundación Lumière / Amigos del Cine.

Dorado, A. (Director) (2004). El rey [Película]. Colombia: Fundación Imagen Latina, Eurocine, C.T.P.

Gaviria, V. (Director) (1988). Rodrigo D. No futuro [Película]. Colombia: Compañía de Fomento Cinematográfico / Focine / Fotoclub-76 / Producciones Tiempos Modernos.

Gaviria, V. (Director) (2004). Sumas y restas [Película]. Colombia: Burn Pictures, Latin Cinema Group, La Ducha Fría Producciones, Latino Films. A.T.P.P. Producciones.

Maillé, E. (Director) (2005). Rosario Tijeras [Película]. Colombia: Coproducción Colombia-México-España-Brasil; Río Negro / United Angels / Dulce Companía / Fondo de Inversión y Estímulos al Cine (Fidecine) / Ibermedia / Maestranza Films / Moonshot Pictures. 